Supporting Information for:

\title{
Large-scale Biomolecular Conformational Transitions Explored by a Combined Elastic Network Model and Enhanced Sampling Molecular Dynamics
}

\section{Conformer generation details in each iteration}

For a given energetically-minimized (in implicit solvent) input structure, the Prody ${ }^{1}$ program was adopted to calculate the Hessian matrix and eigenvectors with CA atoms of the protein (or $\mathrm{P}$ atoms for nucleotides). All atoms were deformed using a displacement vector combined by three lowest-frequency nonzero eigenvectors to sample new conformers along the slowest modes. To balance the conformational coverage and conformer generation speed during the iteration progress, each of the three selected eigenvectors was scaled by a random coefficient from -1 or 1 to form a total of $8\left(2^{3}\right)$ different displacement vectors. It means as many as eight conformers would be produced for one input structure. Besides, to ensure the obtained conformers are physically reliable, each displacement vector was also scaled by a modespecific factor to guarantee the sampled conformers are located within 1.0 $\AA$ RMSD from the given input structure.

Subsequently, these ANM-derived conformers were clustered based on their mutual RMSDs between each other, and the obtained cluster centers (with clustering cutoff $=0.75$ ) were taken as new input structures for following conformational iteration. It should be noted that the clustering cutoff used in this clustering procedure $(0.75 \AA)$ is smaller than that used to estimate the ensemble size of each iteration (2.0 $\AA$ ) in which only conformers with significant variations make sense.

Compared to the original ClustENM ${ }^{2}$ proposed by Doruker et al., our procedure here decreases the number of combinations of the displacement vector in deforming atomic coordinates, thus significantly reduces the computational costs required to minimize the ANMgenerated conformers. Benefitting from this improved speed, we can evaluate the convergence of generated ensembles by increasing the number of iterations. Ultimately, it allows us to explore a broader conformational space with much more iterations (as many as 100 for pdb luwf) whereas only two or five iterations were performed in the work of Doruker. ${ }^{2}$

Concerning the number of modes used to combine the displacement vector, here we selected the three lowest-frequency modes. To show the conformational transition coverage of three slowest modes, we calculated the transition vector between apo (open) and holo (closed) states of $\mathrm{AdK}$, the protein with the most striking conformational changes among three tested systems in this work, and then projected the slowest 30 modes to this transition vector. The cumulative projection indicates the three slowest modes can cover $60 \%-80 \%$ of all conformational changes achieved by 30 lowest-frequency modes, and this proportion could be improved by about $15 \%$ if six slowest modes are used (data not shown). However, eightfold $\left(2^{6} / 2^{3}\right)$ computational demands would be required in energy minimizations to reach the identical rounds of iteration compared to that using three modes. Since the iteration process of iterANM also explicitly integrates the on-going conformational adjustments of the already obtained structures, we think the selection of three slowest modes is more practical for rapidly exploring the conformational transitions of general proteins. For more complicated systems, six or more low-frequency modes, or smarter mode-selection methods should be tested to sample more important 
conformations with higher efficiency.

\section{Simulating details for cMD}

A total of nine functionally diverse proteins were selected and subject to long-time-scaled cMD simulations to test the sampling efficiency of iterANM. Their pdbids and biological functions are listed in Table S1. For each selected protein, the initial structure was generated by removing ligands and waters in the corresponding experimental structure. Atomic cMD simulations were performed in the Gromacs ${ }^{3}$ program with the Amber14SB ${ }^{4}$ force field. Each protein was solvated into a rectangle box of water molecules with at least $1.0 \mathrm{~nm}$ distance from the solute, followed by the addition of $0.15 \mathrm{M} \mathrm{NaCl}$ to neutralize the system. Then these solvated systems were initially minimized and equilibrated by 500 ps NVT and 500 ps NPT MD simulations. To ensure all low-energy conformations are sampled, one $1 \mu$ s and ten independent $100 \mathrm{~ns}$ production runs with NPT ensemble at $300 \mathrm{~K}$ were subsequently performed for each system. Therefore, a total of $2 \mu \mathrm{s}$ MD trajectories were collected for each test protein. During the NPT production run, all bonds involving hydrogen were constrained using the LINCS algorithm to enable an integration timestep of $2 \mathrm{fs}$. The non-bonded interaction was cut off at $1.0 \mathrm{~nm}$, whereas the electrostatic interaction beyond that was treated with particle mesh Ewald (PME) method.

\section{Simulating details for iterANM-IaMD}

In the simulations of $\mathrm{AdK}, \mathrm{CaM}$, and $\mathrm{p} 38 \alpha$, atomic coordinates of apo and ligand-bound proteins were extracted from experimentally determined structures and subject to the iterANM procedure. All mutated residues were modified to relevant wild-typed ones. For ligand-bound structures, the iterANM procedure was performed with the protein atoms, and the ligand was subsequently fitted into the binding pocket of newly generated conformers. Based on the combined apo and ligand-bound ensembles, we selected 7, 8, and 8 high-populated representative conformers for $\mathrm{AdK}, \mathrm{CaM}$, and $\mathrm{p} 38 \alpha$ to define the initial pathway connecting their apo and ligand-bound conformational states.

Before the conformation selection, ensembles generated from apo and holo structures were combined and projected to a two-dimensional PC space to determine the rough pathway connecting the apo and holo conformational states. The representative conformers were selected mainly based on two factors. First, the PCA projections of these structures should be located along the transition path connecting the initial two endpoints. Second, the positions of these structures relative to two endpoints (quantitated by DRMSD) should be evenly distributed. Afterwards, these selected structures were visually checked to ensure they could roughly reflect the structural characterization of the transition path.

Then, two sets of IaMD enhanced sampling simulations (with or without ligand bound) for these representative conformers were carried out. For each representative conformer, two independent IaMD production runs were performed and the simulation lengths of a single trajectory for AdK, CaM, and p38a are 400 ns, 50 ns, and 200 ns, respectively. In IaMD of $\mathrm{AdK}$ and $\mathrm{CaM}$, a boost potential was added to all protein dihedrals to promote conformational sampling, whereas merely dihedrals within the activation loop were boosted for $\mathrm{p} 38 \alpha$ to focus on conformational transitions of this loop.

All IaMD simulations were performed using OpenMM ${ }^{5}$ on GPUs with Amber14SB ${ }^{4}$ force 
field for proteins and $\mathrm{GAFF}^{6}$ for ligands. The simulation workflow of IaMD was same as that adopted in cMD mentioned above except that the hydrogen mass in protein was repartitioned to $4 \mathrm{amu}$ to enable an integration step of $4 \mathrm{fs}$ and all hydrogen-associated bonds of protein were fixed using CCMA algorithm..$^{7-8}$

\section{Trajectory analyses}

The Python packages MDAnalysis, ${ }^{9}$ Prody, ${ }^{1}$ and MsMbuilder ${ }^{10}$ were used to calculate the backbone RMSD, distance between domains, RMSF of CA atoms, and principal components of generated conformers. It should be noted that RMSFs of iterANM-derived conformers were linearly scaled for better comparison with that of MD-derived conformers. The clustering analysis of MD trajectories was performed using the cpptraj module in Amber. ${ }^{11}$ The IaMD snapshots were reweighted by our in-house scripts to construct the free-energy landscape covering the global conformational space. 


\section{Reference:}

1. Bakan, A.; Dutta, A.; Mao, W.; Liu, Y.; Chennubhotla, C.; Lezon, T. R.; Bahar, I. Evol and ProDy for bridging protein sequence evolution and structural dynamics. Bioinformatics 2014, 30 (18), 2681-3.

2. Kurkcuoglu, Z.; Bahar, I.; Doruker, P. ClustENM: ENM-Based Sampling of Essential Conformational Space at Full Atomic Resolution. J. Chem. Theory Comput. 2016, 12 (9), 454962.

3. Abraham, M. J.; Murtola, T.; Schulz, R.; Páll, S.; Smith, J. C.; Hess, B.; Lindahl, E. GROMACS: High performance molecular simulations through multi-level parallelism from laptops to supercomputers. SoftwareX 2015, 1-2, 19-25.

4. Maier, J. A.; Martinez, C.; Kasavajhala, K.; Wickstrom, L.; Hauser, K. E.; Simmerling, C. ff14SB: Improving the Accuracy of Protein Side Chain and Backbone Parameters from ff99SB. J. Chem. Theory Comput. 2015, 11 (8), 3696-713.

5. Eastman, P.; Swails, J.; Chodera, J. D.; McGibbon, R. T.; Zhao, Y.; Beauchamp, K. A.; Wang, L. P.; Simmonett, A. C.; Harrigan, M. P.; Stern, C. D.; Wiewiora, R. P.; Brooks, B. R.; Pande, V. S. OpenMM 7: Rapid development of high performance algorithms for molecular dynamics. PLoS Comput. Biol. 2017, 13 (7), e1005659.

6. Wang, J. M.; Wolf, R. M.; Caldwell, J. W.; Kollman, P. A.; Case, D. A. Development and testing of a general amber force field. J. Comput. Chem. 2004, 25 (9), 1157-1174.

7. Hopkins, C. W.; Le Grand, S.; Walker, R. C.; Roitberg, A. E. Long-Time-Step Molecular Dynamics through Hydrogen Mass Repartitioning. J. Chem. Theory Comput. 2015, 11 (4), 1864-74.

8. Eastman, P.; Pander, V. S. Constant Constraint Matrix Approximation: A Robust, Parallelizable Constraint Method for Molecular Simulations. J. Chem. Theory Comput. 2010, $6(2), 434-437$.

9. Michaud-Agrawal, N.; Denning, E. J.; Woolf, T. B.; Beckstein, O. MDAnalysis: a toolkit for the analysis of molecular dynamics simulations. J. Comput. Chem. 2011, 32 (10), 2319-27. 10. Harrigan, M. P.; Sultan, M. M.; Hernandez, C. X.; Husic, B. E.; Eastman, P.; Schwantes, C. R.; Beauchamp, K. A.; McGibbon, R. T.; Pande, V. S. MSMBuilder: Statistical Models for Biomolecular Dynamics. Biophys. J. 2017, 112 (1), 10-15.

11. Salomon-Ferrer, R.; Case, D. A.; Walker, R. C. An overview of the Amber biomolecular simulation package. WIREs Comput. Mol. Sci. 2013, 3 (2), 198-210. 


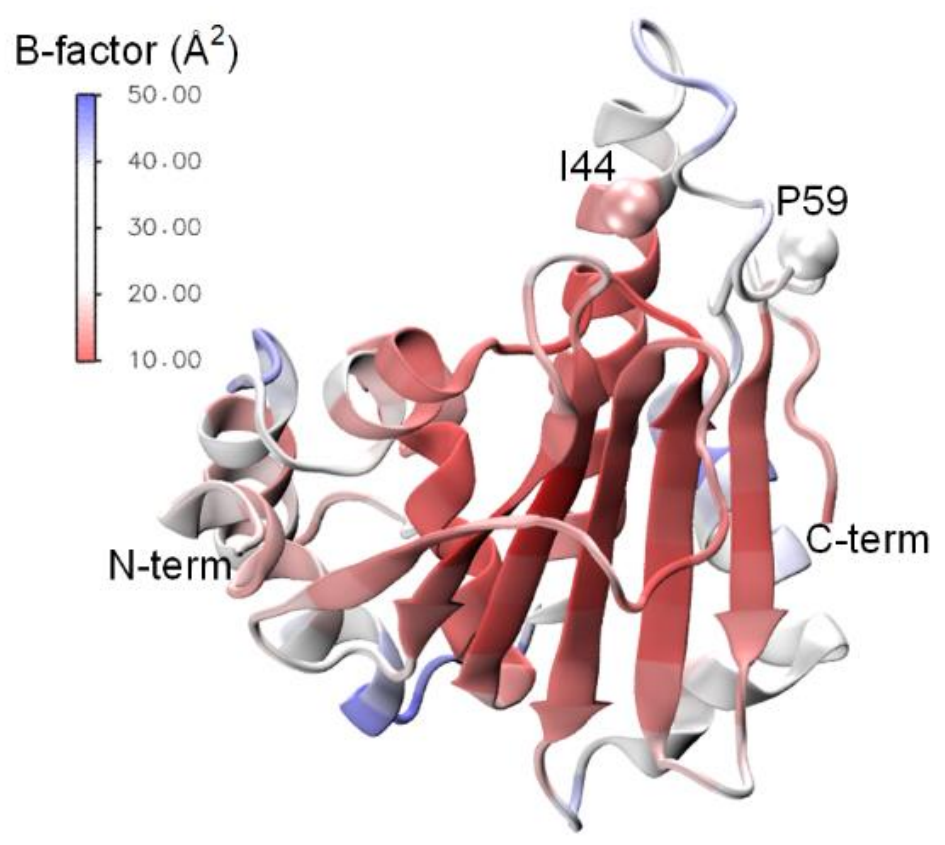

Figure S1. Crystal structure of HSP82 (pdbid 2fxs). The protein is shown in cartoon and colored by B-factors. Residues I44 and P59 are also marked by spheres. To highlight the flexibility within residues 44-59, the $\mathrm{N}$-term and C-term residues are removed.
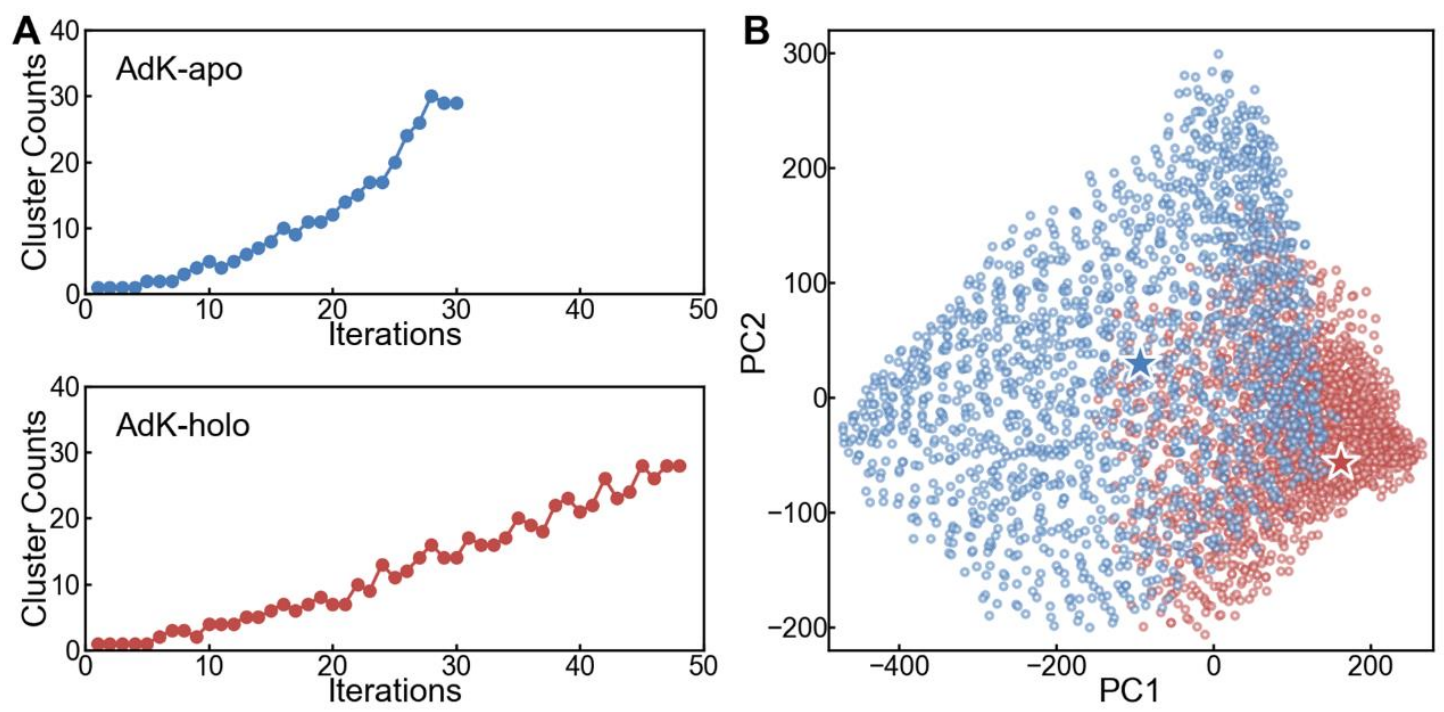

Figure S2. Ensembles of Adk sampled by the iterANM procedure. (A) The cluster numbers of ensembles obtained after each iteration for apo (top panel) and holo (bottom panel) AdK. (B) PCA projections for the ensemble of apo (blue) and holo (red) AdK. The first two PCs could account for $82 \%$ of the conformational variations for AdK. The initial structures of apo and holo states are marked as blue and red stars, respectively. 

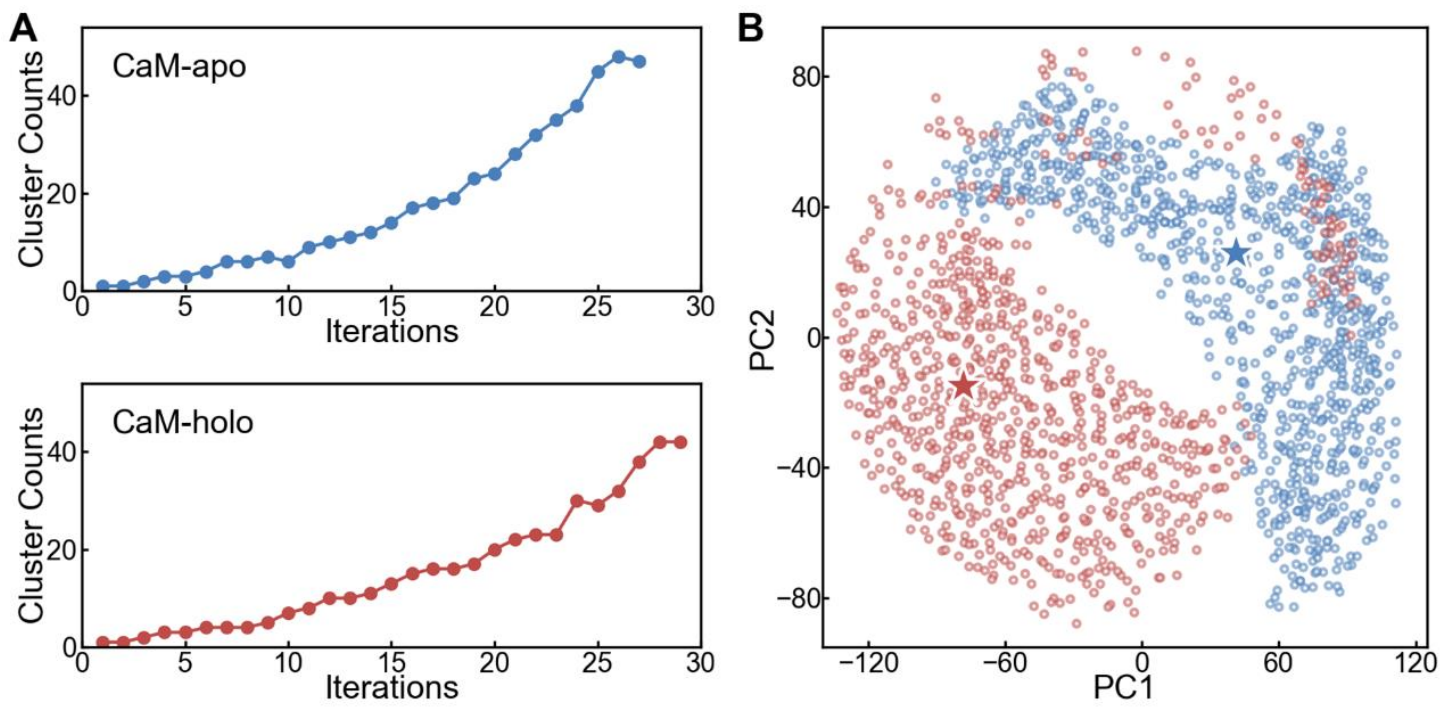

Figure S3. Ensembles of CaM sampled by the iterANM procedure. (A) The cluster numbers of ensembles obtained after each iteration for apo (top panel) and $\mathrm{Ca}^{2+}$-bound (bottom panel) $\mathrm{CaM}$. (B) PCA projections for the ensemble of apo (blue) and $\mathrm{Ca}^{2+}$-bound (red) CaM. The first two PCs could account for $70 \%$ of the conformational variations for CaM. The initial structures of apo and $\mathrm{Ca}^{2+}$-bound states are marked as blue and red stars, respectively.
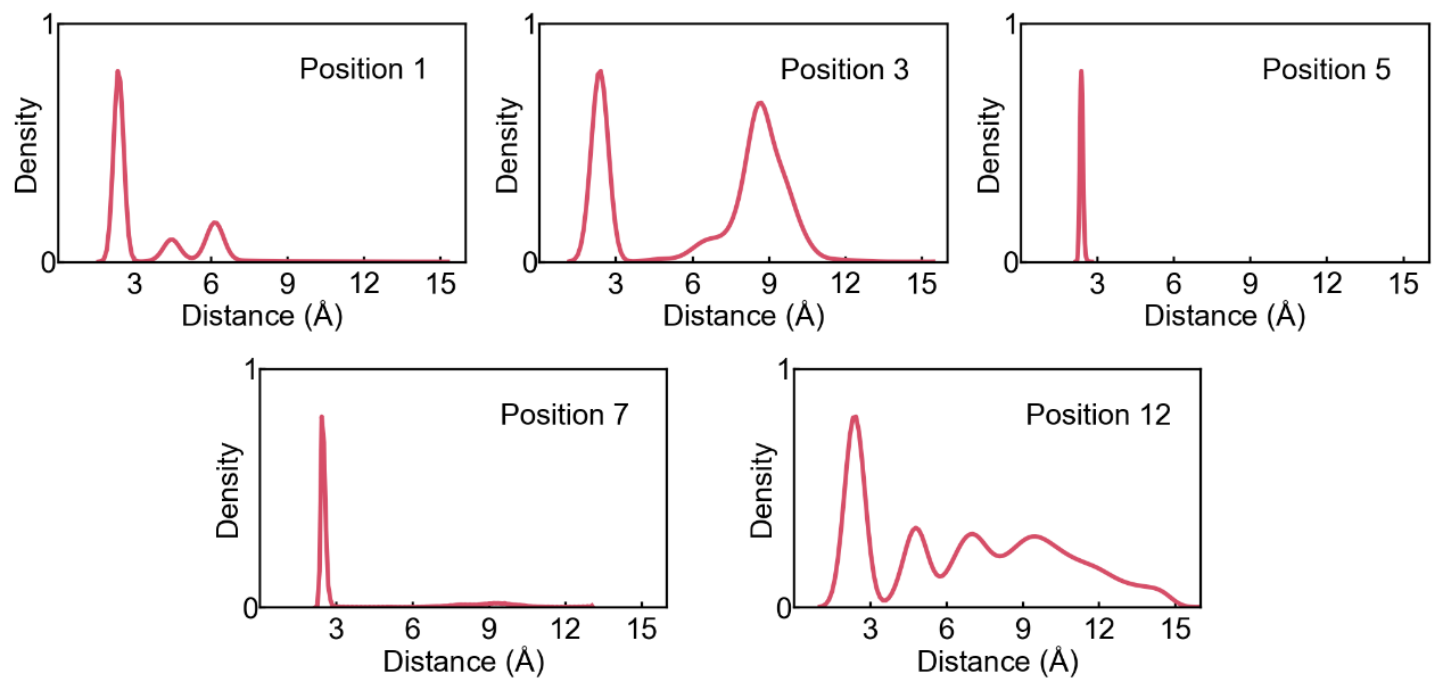

Figure S4. Distributions of the distance between ion and corresponding oxygen atoms of residues involved in coordination with $\mathrm{Ca}^{2+}$ in loop IV of CaM. 

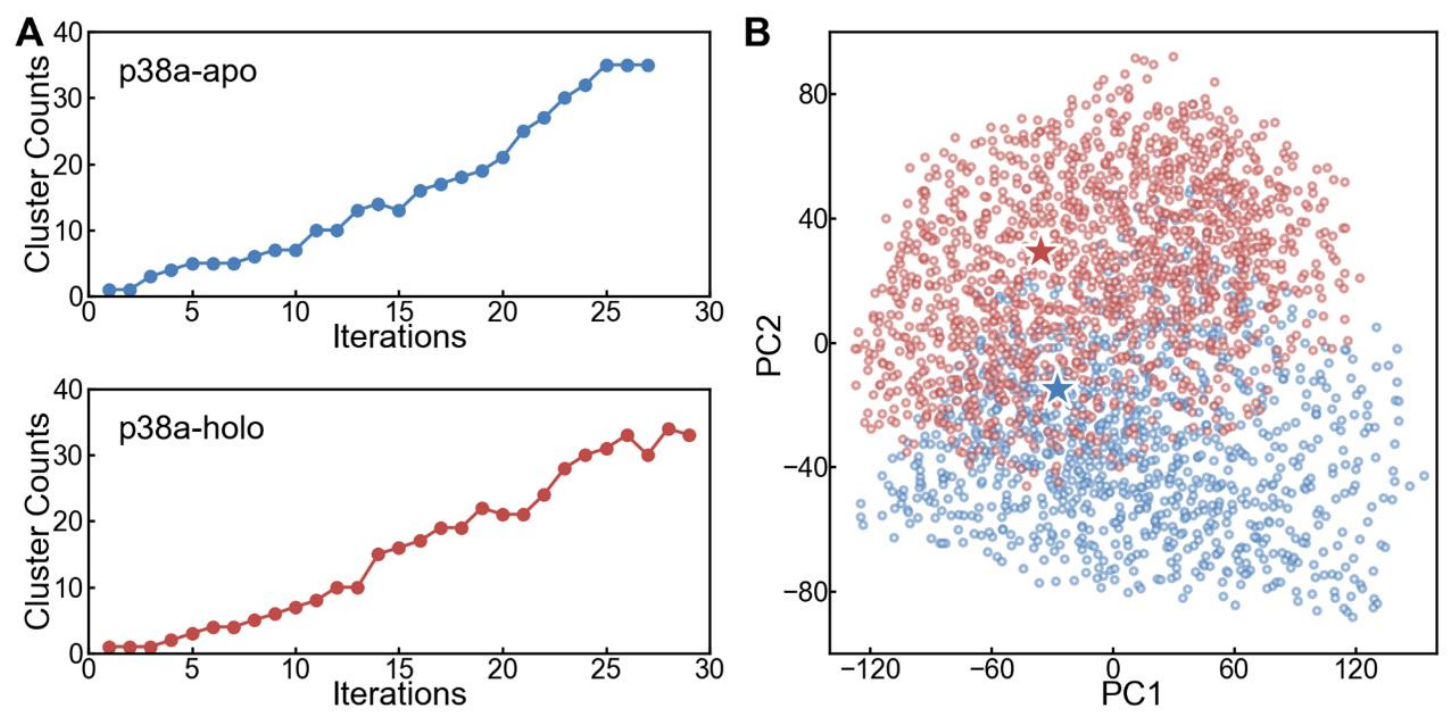

Figure S5. Ensembles of $\mathrm{p} 38 \alpha$ sampled by the iterANM procedure. (A) The cluster numbers of ensembles obtained after each iteration for apo (top panel) and holo (bottom panel) p38a. (B) PCA projections for the ensemble of apo (blue) and holo (red) p38 . The first two PCs could account for $66 \%$ of the conformational variations for $\mathrm{p} 38 \alpha$. The initial structures of apo and holo states are marked as blue and red stars, respectively. 
Table S1. The sequence lengths and biological functions of proteins used to check the conformational space sampling efficiency of iterANM.

\begin{tabular}{|c|c|c|c|c|}
\hline System & PDB & N $_{\text {res }}{ }^{\text {a }}$ & Resolution $(\AA)$ & Biological Functions \\
\hline 1 & 1841 & 162 & 1.8 & Phage lysozyme \\
\hline 2 & $1 \mathrm{~d} 7 \mathrm{i}$ & 107 & 1.9 & FK-506 binding protein (FKBP) \\
\hline 3 & $1 \mathrm{~d} 9 \mathrm{i}$ & 258 & 2.3 & Thrombin \\
\hline 4 & $1 \mathrm{fv} 0$ & 121 & 1.7 & Lysine-,arginine-,ornithine-binding (LAO) protein \\
\hline 5 & $11 \mathrm{af}$ & 238 & 2.1 & Dihydrofolate reductase \\
\hline 6 & $1 \mathrm{u} 71$ & 186 & 2.2 & Mannose-specific adhesin FimH \\
\hline 7 & $1 \mathrm{uwf}$ & 158 & 1.7 & Yeast HSP82 \\
\hline 8 & $2 \mathrm{fxs}$ & 213 & 2.0 & Growth factor receptor-bound protein 2 \\
\hline 9 & 3ove & 101 & 1.8 & \\
\hline
\end{tabular}

${ }^{a}$ Number of amino acids in each tested protein. 